\section{Gerinnungsfaktor VI}

T. Arndt

Bioscientia Institut für Medizinische Diagnostik $\mathrm{GmbH}$, Ingelheim, Deutschland
Definition Historische, heute nicht mehr geführte Nummer aus der Owren-Nummerierung. 